\title{
Möglichkeiten durch E-Sport für Wirtschaft und Gesellschaft
}

\author{
Henri Schmidt, Stefan Lehmann
}

\section{Einleitung}

Dank der permanenten Weiterentwicklung der Mikroelektroniktechnologie gewinnen in der heutigen Gesellschaft Freizeitbeschäftigungen abseits der konventionellen Sportaktivitäten immer mehr an Bedeutung. Mit der Einführung der ersten Spielkonsolen, Ende der sechziger Jahre, begann eine Entwicklung, welche heute, unter anderem, mit dem Namen „E-Sport" bezeichnet wird. Doch was bedeutet E-Sport eigentlich?

„Der Begriff E-Sport (auch: eSport; englisch kurz für electronic sport) bezeichnet das wettbewerbsmäßige Spielen von Computer- oder Videospielen im Mehrspielermodus. E-Sport versteht sich entsprechend der klassischen Definition als eigene Sportdisziplin, welche sowohl Spielkönnen (Hand-Augen-Koordination, Reaktionsschnelligkeit) als auch taktisches Verständnis (Spielübersicht, Spielverständnis) erfordert." [WI05]

Mannschaften werden beim E-Sport als Clans bezeichnet. Solche Clans sind im Allgemeinen hierarchisch organisiert. Ein Spiel zwischen zwei verschiedenen Clans bezeichnet man als Clan War. Solche Clan Wars können entweder in einem lokalen Netzwerk oder über das Internet ausgetragen werden. Clan Wars können entweder nur aus Spaß, zu Trainingszwecken, durchgeführt werden, dann spricht man von Friendly Wars oder aber als normaler Clan War im Ligabetrieb.

Zu den bekanntesten Ligen in Deutschland zählen die ESL (Electronic Sports League), die NGL (Netzstatt Gaming League), die GIGA Liga (Liga des TV-Senders Giga) und die WWCL (World Wide Championship of LAN-Gaming). Während die ersten drei Ligen hauptsächlich über das Internet ausgespielt werden, wird die WWCL, wie der Name schon sagt, ausschließlich auf so genannten „LAN Partys" durchgeführt.

Die Spieler der Topclans in diesen Ligen werden in der Szene als Progamer bezeichnet, um ihre Professionalisierung hervorzuheben. Der Begriff des „Progaming“ stammt urtümlich aus Südkorea. Um das Jahr 1997 herum entwickelten sich dort die ersten Progamerstrukturen, da Breitbandanschlüsse von der Regierung subventioniert und normale Spielkonsolen mit hohen Einfuhrzöllen belegt wurden und so sich die Spieler auf den PC als Spielmedium konzentrierten. Einer der ersten und bekanntesten Spieler dieser Progamerszene war der Spieler des Echtzeitstrategiespiels „Starcraft“ mit dem Namen „Slayers Boxer“. Der Erfolg des Progamings aus Südkorea hatte später auch seine Auswirkungen im amerikanischen und europäischen Raum. Schon im Jahre 1998 kam der erste amerikanische Progamer mit dem Namen „fatal1ty“ („Quake II \& III“) hinzu, welcher sich durch lang anhaltende Siegesserien einen Namen machte. Zahlreiche andere Spieler folgten diesen Vorbildern. Der Durchbruch kam jedoch mit der Veröffentlichung einer Modifikation des Spiels Halflife mit dem Namen "Counter-Strike“, welches einen erheblichen Zuwachs an Onlinespielern verursachte.
Counter-Strike ist ein Egoshooter, in welchem ein Team jeweils das andere Team am Durchführen einer bestimmten Aufgabe zu hindern versucht. $\mathrm{Zu}$ diesen Aufgaben zählen beispielsweise das Legen einer Bombe oder das Befreien von virtuellen Geiseln.

Die Szene in Südkorea ist heute sehr professionalisiert. Progamer werden von Konzernen gesponsort und geniessen ein ähnlich hohes Ansehen wie Popstars. Ist solch ein Erfolg auch hier möglich? Wie weit sind wir in Europa und speziell in Deutschland? Diesen Themen soll diese Arbeit gewidmet sein.

\section{Technische Grundlagen}

Spiele, welche per Internet im Multiplayermodus gespielt werden können, nutzen im Allgemeinen die gleiche Netzwerkstruktur und haben ähnliche Ansprüche an dieses Netzwerk, weshalb an dieser Stelle kurz darauf eingegangen werden soll. Onlinespiele enthalten neben der eigentlichen Spiellogik, der grafischen Benutzeroberfläche auch eine Netzwerkkomponente, welche die Kommunikation über das Internet oder das LAN kontrolliert. Oft entscheidet die Qualität der Netzwerkkomponente, ob ein Spiel online zu einem Erlebnis wird oder nicht. Normalerweise verbindet sich der Rechner des Spielers mit einem eigenständigen Serverprogramm irgendwo im Internet. Alle gängigen Spieletitel verfügen über ein dediziertes Serverprogramm, welches nur die Aufgabe hat, den Netzwerkverkehr zwischen den einzelnen Clientrechnern zu kontrollieren und die Spielsituation zu kontrollieren. Hier wird entschieden, ob Spieler A bspw. den Spieler B getroffen hat oder nicht. Erst nach der Rückmeldung vom Server, wird ein vermeintli-

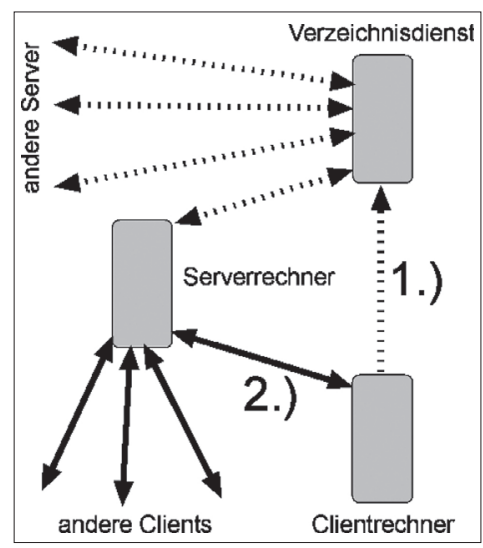

Bild 1: Verzeichnisdienstarchitektur cher Treffer zu einem wirklichen Treffer. Es liegt auf der Hand, dass an dieser Stelle auf dem Clientrechner prediktive Algorithmen zum Einsatz kommen müssen, welche in gewissem Ausmaß Situationen voraus berechnen müssen, damit das Spiel flüssig läuft und keine störenden Pausen aufgrund von nicht vorhandenen Daten auftreten. Das Konzept der dedizierten Serverprogramme erlaubt es, dass das Vermieten von so genannten Gameservern zu einem lukrativen Geschäft werden kann, denn der Serverbetreiber kann je nach Ausstattung seiner PCs, mehrere dedizierte Serverprogramme nebeneinander laufen lassen. Diese Programme 
benötigen nur einen Bruchteil der Rechnerperformance, der eigentlichen Spielprogramme. Zwar haben die meisten Spielprogramme auch einen eigenen Serverpart, aber dieser wird, wenn überhaupt, meist nur auf LANs genutzt.

Um sich mit einem Server zu verbinden, nutzen viele Spieler Servertools (Verzeichnisdienste) wie All-Seeing-Eye, GameSpy oder www.gametiger.net. Dies sind Stand-alone Programme oder Weboberflächen, welche es ermöglichen das Internet nach Servern zu durchsuchen (siehe Bild links, Pfeil 1). Es werden dann, je nach Programm, Informationen über das laufende Spiel und die Serverkonfiguration gegeben.

Ist ein Spieler dann auf einem Server eingeloggt (siehe Bild links, Pfeil 2). So kann das Spiel beginnen. Nun sind der Pingwert und die Bandbreite von größter Wichtigkeit. Der Pingwert beschreibt dabei die Zeit, welches ein Datenpaket zum Server benötigt und zurück. Je geringer diese Zeit ist, desto besser kann man auf bestimmte Ereignisse im Spiel reagieren. Gerade bei Egoshootern ist diese Latenzzeit äußerst wichtig. Mit Fastpath, also dem Ausschalten von Interleaving (1) bei ADSL-Anschlüssen, können Pingzeiten von unter $10 \mathrm{~ms}$ erreicht werden. Pingzeiten, welche größer als 100ms sind, verursachen deutliche Nachteile in der Reaktionsfähigkeit gegenüber den Mitspielern. Die Bandbreite ist ein weiterer Faktor, welcher erhebliche Nachteile im Onlinespiel bringen kann. Je mehr Spieler sich auf einem Server befinden, bzw. je mehr passiert was den Spieler in irgendeiner Weise betrifft, desto mehr Daten müssen übertragen werden. Schlecht implementierte Netzwerkprotokolle haben schon einige Onlinespiele durch dieses Problem unspielbar gemacht, da sie einfach zu viel Traffic verursachten und so bei zu vielen Spielern auf einem Server, bei Klienten mit geringer Bandbreite sogenannte „Lags“ verursachten. „Lags" sind Pausen im Spielfluss die aufgrund fehlender Daten entstehen. Dank DSL tritt dieses Problem heutzutage nicht mehr so häufig auf, da diese Technik mehr als genug Bandbreite für alle gängigen Spiele bereitstellt. Trotzdem ist zu bemerken, dass die Bandbreite den Spielen, in Hinblick auf die maximalen Spielerzahlen auf einem Server, Grenzen setzt. Spiele wie Battlefield 2 unterstützen immerhin bis zu 64 Spieler gleichzeitig auf einem Server. Es gibt Server, welche erheblich mehr Spieler unterstützen, jedoch wenden diese einige Tricks an, so dass im Endeffekt nicht wirklich alle Spieler sich in der gleichen virtuellen „Welt" befinden. Zur Sprachkommunikation werden sogenannte Voicetools eingesetzt, welche auf dem Prinzip der IP-Telefonie basieren.

\section{Vergleich zwischen E-Sport und reellem Sport}

Das Bundesverwaltungsgericht entschied mit einem Urteil vom 9.März 2005, mit folgendem Text: „, [...] Auch der Umstand, dass viele Spiele auch unter Wettbewerbsbedingungen veranstaltet werden können, führt noch nicht dazu, dass aus der Teilnahme am Spiel Sport wird. Computerspiel ist selbst dann kein Sport, wenn es im Wettbewerb veranstaltet wird. Typischerweise wird ein Computerspiel nicht gespielt, um sich zu 'ertüchtigen .[...]" [LI05], dass E-Sport kein Sport ist. Doch kann man solche gesellschaftlichen Fragen vor Gericht klären? Was ist mit etablierten Sportarten, welche auch nicht unbedingt einer körperlichen Ertüchtigung dienen, wie Golf oder Schießsport? Kann man überhaupt Sport nur auf die körperliche Ertüchtigung reduzieren oder gehört nicht viel mehr auch das gesamte Rahmenwerk zum Sport: seine Fans, die Wettkämpfe, die Sponsoren, die Preise, das Nationalbewusstsein und vieles mehr?

Es liegt auf der Hand, dass viele Sportarten eher mit köperlicher Ertüchtigung zu haben als E-Sport und dennoch gibt es viele Gemeinsamkeiten zwischen diesen beiden "Spheren“. Wie auch bei konventionellen Sportarten gab es in den letzten Jahren beim E-Sport einen starken Drang zur Professionalisierung. Es wurde und wird immer schwerer für den Hobbyspieler, sich mit den Besten der Szene zu messen. Dieser Unterschied wird gemeinhin unterschätzt. Heute verbreitete Netzwerkcomputerspiele sind im Allgemeinen so komplex und ausgefeilt in der Art der Nutzerinteraktion und des Spielprinzips, dass es vom Anfänger zum Progamer ein weiter Weg ist. Wie bei traditionellen Sportarten spielen auch bestimmte trainierbare körperliche oder geistige Fertigkeiten eine besondere Rolle. Dazu gehören beispielsweise die Hand-Augen-Koordination, die generelle Reaktionsfähigkeit oder das taktische Verständnis einer bestimmten Spielsituation. Die Frage, ob E-Sport tatsächlich ein Sport ist, kann hier nicht letztendlich geklärt werden, da es zahlreiche verschiedene Definitionen gibt, was Sport sei, bzw. was er nicht sei. Jedoch soll anhand einiger Stichpunkte gezeigt werden, dass E-Sport ähnliche Aspekte besitzt, wie traditioneller Sport:

\begin{tabular}{|l|l|}
\hline Physiologie: & $\begin{array}{l}\text { Reaktionsschnelligkeit, Hand-Augen Koordination, } \\
\text { etc. }\end{array}$ \\
\hline Strukturen: & $\begin{array}{l}\text { Clans, Ligen, Deutscher E-Sport Verband } \\
\text { (www.e-sb.de) }\end{array}$ \\
\hline Wettkämpfe: & Clan Wars, Lan Parties, etc. \\
\hline Medialisierung: & $\begin{array}{l}\text { GlGA eSport sendet mehrmals wöchentlich Neu- } \\
\text { igkeiten, viele verschiedene Webseiten berichten } \\
\text { (bspw.: www.ingame.de) }\end{array}$ \\
\hline Technisierung: & $\begin{array}{l}\text { Profiequipment verfügbar: Grafikkarten, Mäuse, } \\
\text { Pads etc. }\end{array}$ \\
\hline
\end{tabular}

Jedoch gibt es auch einige Problemfelder, in welchen E-Sport gegenüber traditionellen Sportarten noch Aufarbeitungsbedarf hat:

\begin{tabular}{|l|l|}
\hline Psychologie: & $\begin{array}{l}\text { bisher keine gute Vorbereitung auf die } \\
\text { psychischen Strapazen }\end{array}$ \\
\hline Gesellschaft: & $\begin{array}{l}\text { E-Sport wird in der Gesellschaft noch nicht als } \\
\text { Sport wahrgenommen }\end{array}$ \\
\hline
\end{tabular}

\section{Probleme \& Vorurteile}

Neben den schon im Vergleich zwischen E-Sport und traditionellem Sport aufgedeckten Problemfeldern, gibt es einige zusätzliche Probleme, welche die gesellschaftliche Akzeptanz erschweren. Dazu gehört die mittlerweile gängige Praxis der Medien zwischen Gewaltverbrechen aller Art eine Verbindung zum Hobby des Täters herzustellen, welches in einigen Fällen das Computer-Spielen ist. Tatsache ist jedoch, dass bisher keine umfangreichen Studien $\mathrm{zu}$ diesem Thema durchgeführt wurden und allenfalls Mut- 
maßungen zu einer Verbindung zwischen dem Computerspielen und den Taten hergestellt werden können. Nach dem Amoklauf des Schülers in Erfurt waren die schuldigen Faktoren schnell gefunden, doch allzu oft werden allgemeine gesellschaftliche Probleme einfach ausgeblendet und das Problem auf wenige Ursachen reduziert.

Ein weiteres Problem ist, dass in der Bevölkerung ein starkes Bewusstsein besteht, dass viele Spiele zu blutrünstig und somit den Kindern nicht zumutbar seien. Tatsächlich gibt es einige Spiele, die nur Gewaltverherrlichung zum Inhalt haben. Doch solche Spiele setzen sich auf Dauer nicht bei der Masse durch, wie die Onlinespielerzahlen zeigen. Professionelle Spieler verzichten lieber auf die „Extraportion“ Blut, wenn es denn erlaubt ist, da solche Effekte meist eher störend sind und ablenken.

Außerdem gibt es noch die „UnterhaltungssoftwareSelbstkontrolle“ (USK), welche es den Eltern erleichtern soll, die richtige Entscheidung beim Kauf von Spielen für ihre Sprösslinge zu treffen. Softwarefirmen können gegen eine Gebühr ihr Produkt auf die Kinder- und Jugendtauglichkeit prüfen lassen. Die Kennzeichnung eines Softwareprodukts ist seit der Novellierung des Jugendschutzgesetztes im Jahre 2003 verpflichtend. Die Kennzeichnung muss auf der Verpackung des Produkts, sowie auf den entsprechenden Datenträgern deutlich erkennbar sein. Die Spiele werden von unabhängigen Mitarbeitern begutachtet und eingestuft. Die Kennzeichnung der Spiele kann in sechs Stufen erfolgen:

- freigegeben ohne Alterbeschränkung

- freigegeben ab 6 Jahren

- freigegeben ab 12 Jahren

- freigegeben ab 16 Jahren

- keine Jugendfreigabe

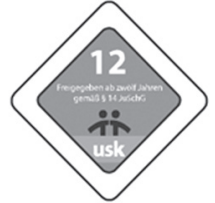

Verweigert jedoch die USK eine Kennzeichnung, bspw. weil ein Spiel den Krieg verherrlicht oder leidende Menschen in einer menschenunwürdigen Situation darstellt (nach $\S 15 \mathrm{Abs} .2 \mathrm{JuSchG}$ ), so ist eine darauf folgende Indizierung durch die Bundesprüfstelle für jugendgefährdende Medien (BPjM) überaus wahrscheinlich. Dass Eltern trotzdem die Blutrünstigkeit der Spiele anprangern, welche ihre Kinder spielen, bedeutet also nur, dass sie sich entweder noch nicht mit der Thematik auseinander gesetzt haben oder dass sie ihrer Fürsorgepflicht nicht nachkommen.

Neben dem Akzeptanzproblem gibt es noch das Glaubwürdigkeitsproblem, welches von vielen Herstellern von Computerspielen bisher unzureichend beachtet wird. Werden Spiele über das Internet ausgetragen, befinden sich die Spieler zumeist an ihrem heimischen Rechner. Wie jede Software sind auch Spiele durch andere „bösartige“ Software „knackbar“. Je populärer ein Spiel wird, desto größer wird die Anzahl der Spieler, welche sich durch das Anwenden sogenannter Cheatprogramme einen Vorteil verschaffen wollen. So können Spieler bspw. durch das Anwenden eines „Cheats" die Wände ausblenden („Wallhack“), die Gegner automatisch anvisieren („Aimbot“) oder sich sonstige Vorteile verschaffende Informationen anzeigen lassen. Die Anzahl der verfügbaren „Cheats“ variiert dabei meist nach den implementierten Sicherheitsvorkehrungen des Spielherstellers solchen „Cheats" vorzubeugen und der Verbreitung des jeweiligen Spiels. Denn auch hier gilt: die Nachfrage bestimmt das Angebot. Doch zumindest im professionellen Bereich kann es sich ein Spieler nicht leisten, einfach auf „Cheats“ zurückzugreifen. Viel zu oft muss er die Wettkampfsituation auf einer öffentlichen Veranstaltung bestreiten. Schummeln ist dort so gut wie unmöglich. Durch die Probleme in der Vergangenheit haben die Hersteller dazu gelernt und so enthält nahezu jedes neue bekanntere Onlinespiel einen Anticheatalgorithmus, welcher das Schummeln erschweren soll.

Ein weiteres Problem ist die mangelnde Bewegung bei allen Arten von Computerspielen. Das ausdauernde Verharren in einer Sitzposition wirkt sich auf die Dauer negativ auf die Gesamtkonstitution des Menschen aus. Auch Haltungsschäden resultieren oft aus dem langwierigen Sitzen. Für die Augen ist das andauernde Fokussieren auf den relativ nahen Monitor anstrengend und ermüdend. Es bedarf einer umfangreichen Aufklärung, dass die Spieler das Bewusstsein erlangen, eine Ausgleichssportart zu betreiben. Für das Sitzen am PC gibt es eine Reihe von Übungen und Hilfsmitteln, welche das Arbeiten und Spielen daran, auf die Dauer „gesünder“ machen. Zu den Hilfsmitteln gehören bspw. ergonomische Eingabegeräte, strahlungsarme Monitore und eine vorteilhafte Positionierung der Peripheriegeräte, denn es gibt bestimmte Winkel und Entfernungen, in welchem die Geräte besonders günstig stehen.

\section{E-Sport in Südkorea - ein etablierter Markt}

Trotz allen Problemen und Vorurteilen, gibt es ein Land, in welchem E-Sport ein mittlerweile anerkannter sportlicher Event ist. Die Akteure haben den gleichen Bekanntheitsgrad wie Popstars. Es existieren dort schon drei Fernsehsender, welche ausschließlich über das beliebte Echtzeitstrategiespiel „Starcraft“ berichten. Das bekannte „Team-Intel“ bekommt ein monatliches Gehalt gezahlt und Ablösesummen für Spielerwechsel zu zahlen ist durchaus nichts Ungewöhnliches mehr. Genauso normal ist es, dass professionelle Clans in Südkorea einen oder mehrere finanzkräftige Sponsoringpartner hinter sich zu stehen haben, welche die Clans mit Equipment oder auch monetär unterstützen, denn es lohnt sich. Der hohe Bekanntheitsgrad der Spieler in den entsprechenden Zielgruppen macht das Geschäft für beide Sponsoringpartner sehr attraktiv.

Um zum Profispieler zu werden ist die Motivation für viele Jugendliche besonders groß, sich längerfristig mit einem bestimmten Spiel zu beschäftigen, um in diesem einen Spiel immer besser zu werden. Zudem locken hohe Preisgelder und Gehälter viele Jugendliche, den Beruf des Profispielers anzustreben. Diese Situation hat auch jedoch ihre Schattenseiten. Man geht davon aus, dass bereits ca. 10\% [WI205] der Jugendlichen Südkoreas süchtig nach dem Internet sind.

Schon heute werden regelmäßig Spiele zwischen den „Helden“ der hiesigen Szene in richtigen Fußballstadien durchgeführt. An einem einzigen E-Sport Event nehmen bis zu 100.000 Menschen teil.

Im Dezember 2004 entschloss sich das dortige Ministerium für Kultur und Tourismus dazu, Pläne für ein eigenständiges E-Sportstadium zu prüfen, um Südkorea damit zum Vorreiter in der Welt zu machen. [KR05] 


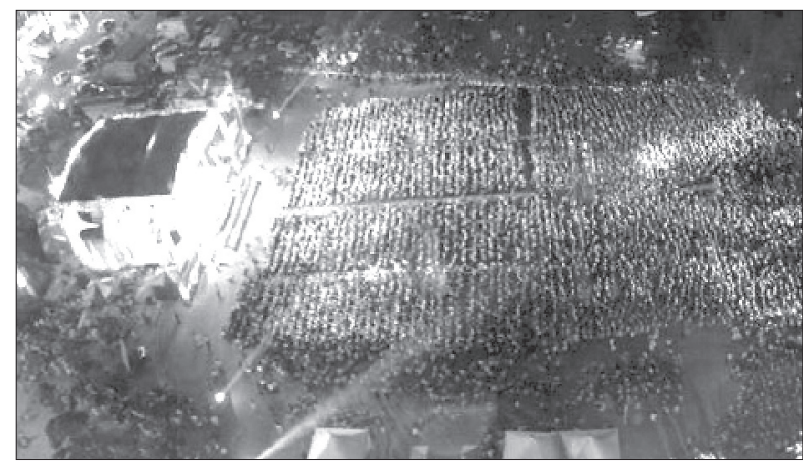

Bild 3: Bis zu 100.000 Menschen bei einem einzigen E-Sport Event in Südkorea

Der weltweit größte E-Sport Event: die World Cyber Games (WCG), fand bisher dreimal in Südkorea statt. Zweimal in Seoul (2001 \& 2003) und einmal in Daejeon (2002). Bei den WCG 2005 werden Preise von insgesamt über 400.000\$ ausgespielt. Damit ist das WCG zwar nicht das höchstdotierte, aber bei weitem das prestigeträchtigste Turnier auf der ganzen Welt, da über 60 Nationen daran teilnehmen. Über eine Million Spieler nahmen 2004 an den Vorrunden zur WCG teil und hatten ca. 2,5 Millionen Zuschauer. Ähnlich wie bei den olympischen Spielen werden die Besten der Besten in einer zwölfmonatigen Qualifikationsphase ermittelt. Am Ende dieser Qualifikationsphase steht ein nationales Finale. Die Sieger dieses Finales bilden die Nationalmannschaft eines Landes und fahren zu den WCG. Im Jahre 2003 errang die deutsche Nationalmannschaft mit dreimal "Gold“ und zweimal „Silber" den Weltmeistertitel. Die globale Organisation der gesamten Spiele obliegt bis heute Südkorea. Die WCG 2005 werden in Singapur, also wieder im asiatischen Raum, stattfinden. Innerhalb von 5 Jahren erreichten die WCG eine Anzahl von teilnehmenden Ländern, wie die Fußballweltmeisterschaft und die olympischen Spiele erst in über 50 Jahren erreichten.

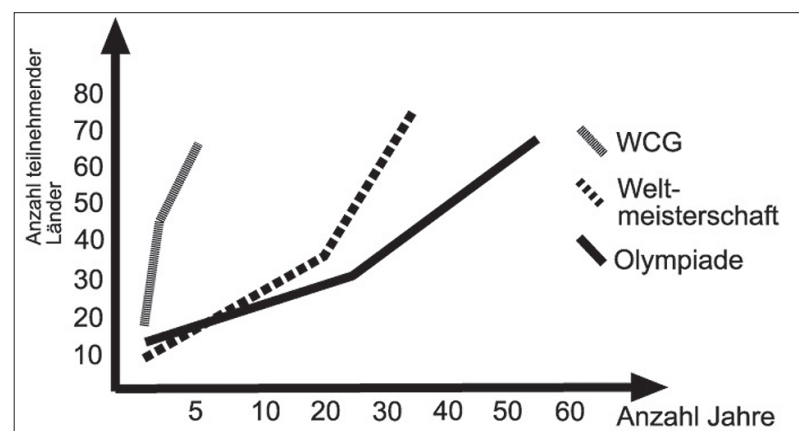

Bild 4: Länderteilnahme an sportlichen Wettbewerben

Wie auch die traditionelle Sportszene, so ist auch die ESportszene immerwährenden Veränderungen unterworfen, so findet bspw. zurzeit in Südkorea ein Wechsel, vom lange Zeit favorisierten, aber technisch altbackenen "Starcraft", hin zum neueren "Warcraft 3“ statt, welches ebenso ein Echtzeitstrategiespiel ist.

\section{E-Sport national und international}

Südkorea ist der restlichen Welt in Sachen „E-Sport" um einiges voraus. Doch Jugendliche aus Amerika und Europa eifern ihren Vorbildern in Südkorea nach und fahren zum Teil selbst nach Südkorea, um dort mit ihrem „Hobby“ Geld zu verdienen. Zwar werden Progaming-Clans auch in der restlichen Welt von Sponsoren unterstützt, aber nicht in dem Umfang wie in Südkorea. Die fortschreitende Professionalisierung kann an der zunehmenden Anzahl von eigenständigen Clans mit ausgefeilten Sponsorenkonzepten gesehen werden, welche teilweise richtige Fan-Communities unter sich vereinen. Die Tatsache, dass E-Sport größtenteils auf der Internettechnologie basiert, macht den Fankontakt einfacher. Die Communities wachsen viel schneller als bei traditionellen Sportarten.

Es treffen sich täglich mehrere 100.000 Spieler auf den Servern weltweit, um gegeneinander die unterschiedlichsten Spiele zu spielen.

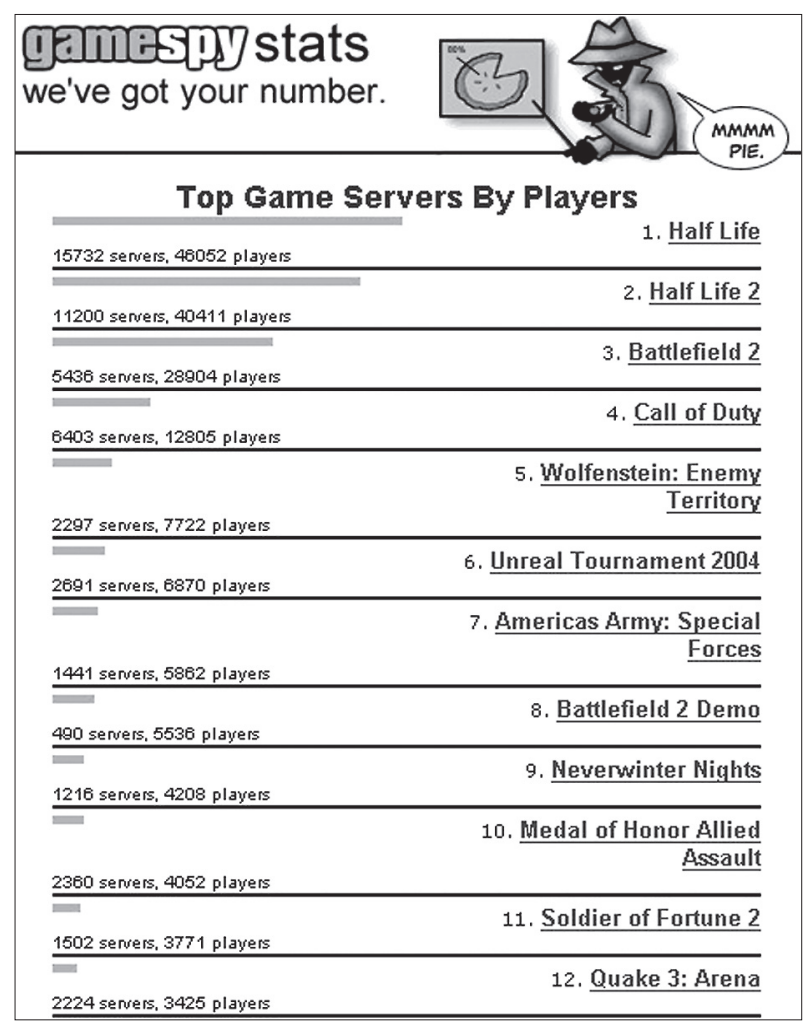

Bild 5: Serverstats von gamespy (30. Juni 2005, 20 Uhr)

Die Serverstats von „gamespy“ (www.gamespy.com) zeigen die deutliche Dominanz der Egoshooter im Onlinebereich. Bis auf „Neverwinter Nights" entsprechen alle hier aufgelisteten Titel diesem Genre. Während in Südkorea vorwiegend Echtzeitstrategiespiele die E-Sportszene bestimmen, sind es im restlichen Teil der Welt vor allen Dingen Egoshooter, welche von der E-Sportszene favorisiert werden.

Zahlreiche Onlinemagazine widmen sich mittlerweile dem Thema „E-Sport" und mit „Giga eSports" ging unlängst auch die erste reine E-Sport TV Sendung "on air“, welche ausschließlich über Neuigkeiten und Trends in der E-Sportszene berichtet. Viele verschiedene Webangebote bieten Livereportagen und Analysen von Clan Wars an. Zudem informieren sie über Neuigkeiten, bspw. Updates oder Patches der Spielesoftware und andere relevante Fakten. Als Beispiele seien hier die beiden E-Sport Portale: www.ingame.de und www.gamelinks.de genannt, welche sich auf zahlreichen Unterseiten mit zahlreichen aktuellen Onlinespieletiteln befassen. 
Besonders gut lässt sich die zunehmende Unterstützung der Bevölkerung und die damit zunehmende Akzeptanz des E-Sports auch an den Unterstützungen staatlicher Institutionen für die World Cyber Games ablesen.

\begin{tabular}{|c|c|}
\hline Countiv & Qepartinemb \\
\hline \multirow{4}{*}{ Argentina } & Sports Committee of National Serate \\
\hline & National Olympic Committee \\
\hline & Buenos Aires city government \\
\hline & Federal Government \\
\hline \multirow{2}{*}{ Brazil } & Federal Government \\
\hline & Brasilia State Government \\
\hline Bulgaria & Ministry of Youth \& Sports \\
\hline Civile . & Ministry of National Defense \\
\hline China & Ministry of Culture \\
\hline $\begin{array}{l}\text { Chinese } \\
\text { Taipei }\end{array}$ & Taipel City - Department of Information \\
\hline Colombia & Korea Embassy \\
\hline Ecuador & National Olympic Committee \\
\hline Estonia & National Olympic Committee \\
\hline Issrael & Tel Aviv city government \\
\hline Korea & Ministry of Culture and Tourism, Daegu City \\
\hline Lithuania & Lithusanian Olympic Foundation \\
\hline Comery & 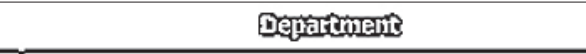 \\
\hline Malaysia & Ministry of Youth \& Sports Malaysia \\
\hline \multirow{3}{*}{ Panama } & E-Commerce Bureau of National Government \\
\hline & Panama City Government \\
\hline & National Olympic Committee \\
\hline Philippines & Philippines Sports Commlssion \\
\hline \multirow{2}{*}{ Portugal } & Porto City Government \\
\hline & National Olympic Committee \\
\hline \multirow{2}{*}{ Russia } & Ministry of Education \\
\hline & Moscow Sport Committee \\
\hline $\begin{array}{l}\text { Serbia \& } \\
\text { Montenegro }\end{array}$ & Belgrade City Government \\
\hline \multirow{3}{*}{ Singapore } & $\begin{array}{l}\text { Ministry of Ynformation, Communications \& the } \\
\text { Atts }\end{array}$ \\
\hline & $\begin{array}{l}\text { Ministry of Community Development, Youth \& } \\
\text { Sppits }\end{array}$ \\
\hline & Economlc Development Board of Singapore \\
\hline Thailant & Mịistry of Information and Communication \\
\hline Uzhekistan & $\begin{array}{l}\text { Uzbeklstin Agency of Communlcalions \& } \\
\text { Information }\end{array}$ \\
\hline Vietnam & $\begin{array}{l}\text { Ho Chi Minh City - Cultural \& Information } \\
\text { Department }\end{array}$ \\
\hline
\end{tabular}

Bild 6: Öffentliche Institutionen, welche die Teams der jeweiligen Länder bei der Teilnahme zur WCG 2004 unterstützten

Bemerkenswert ist hierbei, dass das deutsche Team trotz des Weltmeistertitels aus dem Jahre 2003 scheinbar keinerlei Unterstützung aus öffentlichen Mitteln erhielt. Außerdem scheint die Frage, ob es sich bei E-Sport um wirklichen Sport handelt, in vielen Ländern schon geklärt zu haben, da man erkennen kann, dass es vielerlei Unterstützung von Sportministerien gab. Ein weiteres Indiz dafür, dass man solche Fragen nicht juristisch klären kann, sondern ein rein gesellschaftliches Problem ist.

Bemerkenswert ist auch der Sieg eines 21-jährigen Chinesen im November 2004 beim E-Sportevent „ACON Fatal1ty Shootout" an der chinesischen Mauer. Der junge Chinese Meng Yang bezwang den Progamerstar „Fatal1ty“ aus Amerika, welcher bis dahin als der unbezwungene Champion des Egoshooters „Quake III“ galt, und gewann damit den höchsten Einzelpreis, welcher jemals in der Geschichte des E-Sports ausgespielt wurde. Das Preisgeld von $125.000 \$$ entspricht ca. 1.000 .000 chinesischen Renminbim, wofür ein chinesischer Arbeiter im Durchschnitt 100 Jahre arbeiten muss. Yang sagte zu seinem Sieg: „[...] I practiced hard to play in this exhibition because I wanted to play my idol ... I've studied him for 5 years and dreamed of playing the number one gamer in the world [...]" [FAT05]

Mit der Gründung des „Deutschen eSport Bundes” (ESB) am 11.12.2004 schlossen sich die beiden separaten Verbände, DeSpV und DeSV, zusammen, um gemeinsam den deutschen E-Sport gegenüber der Öffentlichkeit und der Politik adäquat zu repräsentieren. Die Mitgliedschaft an diesem Verband steht jeder Person offen, welche sich für den E-Sport einsetzen möchte. Der Verband wird seit der Gründung von Repräsentanten der Wirtschaft, wie beispielsweise „Intel”, „ATI" und „Shuttle”, unterstützt und arbeitet eng mit der USK (UnterhaltungssoftwareSelbstkontrolle) zusammen. Damit ist gewährleistet, dass die E-Sportszene in Deutschland sich in Zukunft auf einen soliden Rahmenverband verlassen kann, welcher als Mittler zwischen E-Sportszene und der Wirtschaft fungieren kann. Man kann gut erkennen, dass sich der E-Sport langsam etabliert.

\section{Gesellschaftliche Auswirkungen des E-Sports}

Vor allem Jugendliche sind von den Auswirkungen des ESports betroffen. Es entstehen völlig neue Strukturen, wie virtuelle Communities oder Freundeskreise. Oft kommt es vor, dass zwei Spieler eines Clans sich nie persönlich treffen, da sie in zwei unterschiedlichen Ländern oder einfach weit voneinander entfernt wohnen. Das Internet als Grundlage der Kommunikation löst die geografische Abhängigkeit einer Sportmannschaft auf. Es entstehen neue Verbindungen zwischen verschiedenen Nationalitäten, die vorher vielleicht unmöglich erschienen.

Dass Menschen eventuell einen größeren virtuellen Freundeskreis, als einen realen Freundeskreis haben, wirft neue Fragen auf. Inwiefern sind solche Freundschaften mit realen Freundschaften vergleichbar? Was ist mit Menschen, die nur noch virtuelle Freunde haben? Diese Abhängigkeit vom Internet schafft Platz für Gefahren, wie der Sucht nach dem Internet. In Südkorea wurde dieses Problem schon erkannt, jedoch fehlen momentan noch Lösungsansätze für dieses Problem.

Für gesellschaftliche Randgruppen birgt der E-Sport jedoch auch große Chancen. Denn die gesamtkörperliche Verfassung ist bei vielen Onlinespielen relativ egal und somit haben körperlich Behinderte unter Umständen die 
Möglichkeit einen Sport auszuüben und Erfolgserlebnisse zu erfahren, welche ihnen sonst verwehrt blieben.

Die Welt wird zwar auf der einen Seite zusammen wachsen, da körperliche, geografische oder nationale Unterschiede schwinden. Im Gegensatz dazu wächst jedoch unter Umständen der Verlust des persönlichen Kontakts zu anderen Menschen in der Realwelt. Jedoch muss man hier fairer Weise erwähnen, dass gerade bei erhöhtem Professionalisierungsgrad der persönliche Kontakt in der Realwelt eher steigt, da die größeren Veranstaltungen, wie das WCG Finale oder die WWCL lokal ausgetragen werden.

Auch für den Arbeitsmarkt bietet der E-Sport völlig neue Perspektiven. Es entstehen Berufe, wie es sie vorher nicht gab, da das Organisieren, Durchführen und Verwalten von E-Sport-Events völlig andere Anforderungen an die Auszuführenden stellt als traditionelle Sportarten. So haben sich bspw. bei professionellen E-Sport Clans mehrere Aufgabenbereiche herauskristallisiert:

\begin{tabular}{|l|l|}
\hline Spieler: & Spielen von Clan Wars \\
\hline Organisatoren: & $\begin{array}{l}\text { Vorbereiten \& Durchführen von Clan Wars, } \\
\text { LANs, etc. }\end{array}$ \\
\hline Redaktionell: & $\begin{array}{l}\text { Betreuen der redaktionellen Inhalte der Clan- } \\
\text { homepage }\end{array}$ \\
\hline Web: & Erstellen und Warten der Clanhomepage \\
\hline Live: & Durchführen von Livereportagen via IRC, Radio, TV \\
\hline Forum: & Betreuung der Fan-Community \\
\hline Video: & Erstellen und Bearbeiten von Videos über den Clan \\
\hline
\end{tabular}

Dies zeigt, dass es verschiedene Möglichkeiten im E-Sport gibt, beruflich tätig zu werden und nicht alle haben mit bloßem Spielen zu tun. Auch abseits der Clans entsteht Bedarf für entsprechende Fachkräfte. Mit zunehmender Größe und Häufigkeit von E-Sport Events wächst die Notwendigkeit von Fachleuten mit entsprechendem Netzwerkwissen. Auch Promotionexperten, Grafikdesigner und Betriebswirtschaftler werden benötigt, um entsprechende Projekte, wie LAN Parties etc., durchzuführen. Die erste Generation von Onlinespielern dringt nun langsam in die Arbeitswelt der ehemals Erwachsenen ein. Sie haben einen ganz anderen Blickwinkel auf diese Thematik, als die etablierte Generation, da sie selbst Teil der E-Sportszene waren oder zumindest verstehen, um was es dabei geht. Diese Durchdringung wird wahrscheinlich langfristig die Akzeptanz erhöhen und den E-Sport zu einer etablierten Sportart neben den traditionellen Sportarten machen.

Clans haben ähnliche Auswirkungen auf den Spieler wie traditionelle Sportvereine. Durch die hierarchische Organisationsstruktur wird den Spielern eine Teamfähigkeit vermittelt. Das gemeinsame Erarbeiten von Taktiken und der sprachliche Austausch während des Clan Wars machen die Spieler kommunikativer, da sie lernen müssen, wichtige Inhalte in kurzer Zeit zusammenzufassen, die Taktik zu bereden usw.. Außerdem lernen die Spieler auch, konstruktiv mit Kritik umzugehen, denn nur wer Ratschläge annimmt, ist in der Lage sich weiterzuentwickeln.

Auch die Eltern von morgen werden wahrscheinlich bewusster mit dieser Thematik umgehen, da sie mit den Möglichkeiten und Problemen mehr anzufangen wissen, als die heutige Elterngeneration.

Schon im frühen Alter werden Kinder heutzutage mit Telekommunikationstechnologie in Verbindung gebracht.
Das Handy wird zu einem Gebrauchsgegenstand, genauso wie es die Kommunikation und das Spielen über das Internet wird. Inwieweit dies die Gesellschaft konkret verändern wird, lässt sich schwer abschätzen.

\section{Möglichkeiten für die Wirtschaft (Sponsoring)}

E-Sport hat das Potential sich zu einer Trendsportart zu entwickeln. Die Tatsache, dass hier ein Markt mit ausgeprägten Chancen und Risiken wartet und dass bereits erste ökonomische Erfolge gefeiert werden konnten, hat auch Marketingführer von Topfirmen und Pressevertreter aufmerksam werden lassen. Zu diesem Zweck rief Frank Sliwka, geschäftsführendes Vorstandsmitglied des deutschen eSport Bundes, eine Veranstaltung ins Leben, in der sowohl Geschäftsleute der E-Sport Szene, Presse, Firmen wie auch ganz normale Besucher eine Diskussion zu diesem Thema führen können.

Die „Internationales eSport Forum 2005“ genannte Veranstaltung fand im Juni 2005 im Congress Center Düsseldorf (CCD) statt und konnte mit Referenten aus der Wirtschaft und Wissenschaft aufwarten. Unter Anderem, war ein Vortrag von Don S. Kim (Managing Director von AC Entertainment und Unternehmensmitglied von Samsung) zu hören der sich mit dem Thema "Why a big company like Samsung is sponsoring eSport events" [KI05] befasste. Er führte als Beispiel für E-Sport Marketing, eine der größten E-Sportveranstaltungen weltweit, die World Cyber Games an. Er stellte dar, dass ein Event mit 1 Millionen Spielern und 2,5 Millionen Zuschauern weltweit in 63 Ländern die beste Möglichkeit sei, um Marketingerfolge in einer Zielgruppe zu erreichen.

Spiele sind das Medium der Zukunft. Sie beinhalten Unterhaltung in Form von Musik, Filmen, Grafik, Story, dem Spiel an sich und Wettbewerb. Sie berühren auch gesellschaftliche Aspekte der Zukunft wie Kommunikation über Netzwerke oder MMORPGs (Massivly Multiplayer Online Role Playing Games). Es scheint sich herauszukristallisieren, dass Spieler eine dankbare Zielgruppe darstellen: sie passen sich schnell an und liefern Meinungen über neue Produkte, haben Einfluss auf den Kauf von IT Technologien und zählen zu den „Power Buyers“, die mehr kaufen und mehr bezahlen. Laut Don S. Kim ist E-Sport Sponsoring eines der kosteneffektivsten Marketinginstrumente.

Des Weiteren erreicht dadurch die Wirtschaft die junge Generation. Die Kultur ist international und zeigt ein starkes Wachstum, der Geist von E-Sport vermittelt Menschlichkeit, Freundschaft und Fairplay, das Image erscheint fortgeschritten, hoch technisiert und führend. Dadurch besteht beim Zielpublikum laut Kim die Möglichkeit, eine dauerhafte Markenloyalität zu erreichen.

Aus einem Vortrag von Philip Militz (Managing Director Scoopcom!) geht hervor, dass der typische Spieler ein Alter zwischen 14 und 29 Jahren besitzt, zu 97\% männlich ist und 20\% der Spieler über $500 €$ im Monat für „Luxusartikel" zur Verfügung hat, $20 \%$ über $250 €$ und weitere $24 \%$ über $100 €$. $60 \%$ stimmten der Aussage zu „Probiere immer wieder gerne Neues" und 76\% der Aussage „Kauf von Markenartikeln lohnt sich“. [MI05] 
Um in Deutschland einen ähnlichen Markterfolg wie in den asiatischen Ländern mit E-Sport zu erreichen, müssen erst die Vorraussetzungen dafür geschaffen werden, so Christian Sauer vom Clan „OCRANA“, der international vertreten ist und einer der ältesten Clans in Deutschland. Es müsse eine Verbesserung der Wahrnehmung von E-Sport innerhalb der Gesellschaft geschaffen werden, konsequente Orientierung an den Bedürfnissen und Wünschen der Zielgruppen, vereinfachter inhaltlicher und technischer Einstieg in den aktiven E-Sport und die Schaffung zuschauertauglicher und -freundlicher Formate angestrebt werden. Auch wäre die Schaffung von Identifikationsmöglichkeiten durch Stars und Marken, die Darstellung des E-Sport als geeignete Kommunikationsplattform auch für traditionelle Unternehmen, die Etablierung eines E-Sport-Verbandes, sowie eine Konsolidierung der Ligenvielfalt und die Marktforschung mit Wertdarstellung der Rechte der richtige Weg. (1. Juli 2005, http: //www.esportforum.com/vortraege/Vortrag_Sauer.pdf)

Zur Entwicklung des E-Sport Sponsoring meint Rene Korte (Managing Director Gamers Wear and mtw), dass 1998 E-Sport noch ein sponsorenfreier Raum und kommerzfeindlich war und dagegen 2005 über 100 Sponsoren aus der Wirtschaft mit hoher Vertriebs- und Brandingwirkung vorhanden sind. Allerdings sei es bis heute nicht geschafft worden, die großen Marken außerhalb der Technikindustrie zu überzeugen. Er sieht jedoch E-Sport als eine wirtschaftstreibende Kraft mit Beschäftigungsperspektiven an und meint, E-Sport habe „[...] in den letzen Jahren zu einem echten Gründerboom geführt, fern jeder Kapitalismus und Heuschrecken Diskussion!". [KO05]

Jedoch fehle weiterhin professionelle Unterstützung und Mut zur Bereitstellung von Kapital.

Die Chancen des Sponsoring sieht Rene Korte in der Tatsache, dass E-Sport interaktive Marketingmöglichkeiten mit direktem Feedback und Input für Produkte und Produktentwicklungen bietet. "Gaming is a global language" [KO205] eröffnet die Möglichkeit weltweit einen Markt zu erschließen.

Die Risiken des Sponsoring lägen im fehlenden Vertrauen gegenüber des E-Sports und seinen Organisationen und der hohen Anforderung an Professionalität, welche aber teilweise aufgrund fehlender monetärer Mittel nicht erfüllt werden kann. Die Unternehmen würden die Beschädigung der eigenen Marke durch öffentliche Diskussionen fürchten. Der Weg zur Besserung wäre die Aufklärung im eigenen Unternehmen und Verständnis für soziale Umstände.

\section{Auswertung des Fragebogens}

Um aus erster Hand an Statistiken und Meinungen von Spielern zu kommen, wurde ein Fragebogen im Internet zugänglich gemacht. Er richtete sich hauptsächlich an diejenigen Internetbenutzer, die bereits Kontakt mit Online Games oder eSport hatten. Der Fragebogen war in Form eines im Internet üblichen Formulars gehalten, die Antworten waren meist vorgegeben und konnten aus einer vorhandenen Liste ausgewählt werden und in Einzelfällen hatte der Befragte die Möglichkeit, selbst Text frei einzugeben. Die Befragung war in den Sprachen Deutsch und Englisch verfügbar um auch internationalen Interessenten die Möglichkeit der Teilnahme zu geben. Über einen InfoButton hatte der Befragte die Möglichkeit, sich über die Motivation und Urheber der Umfrage zu informieren.

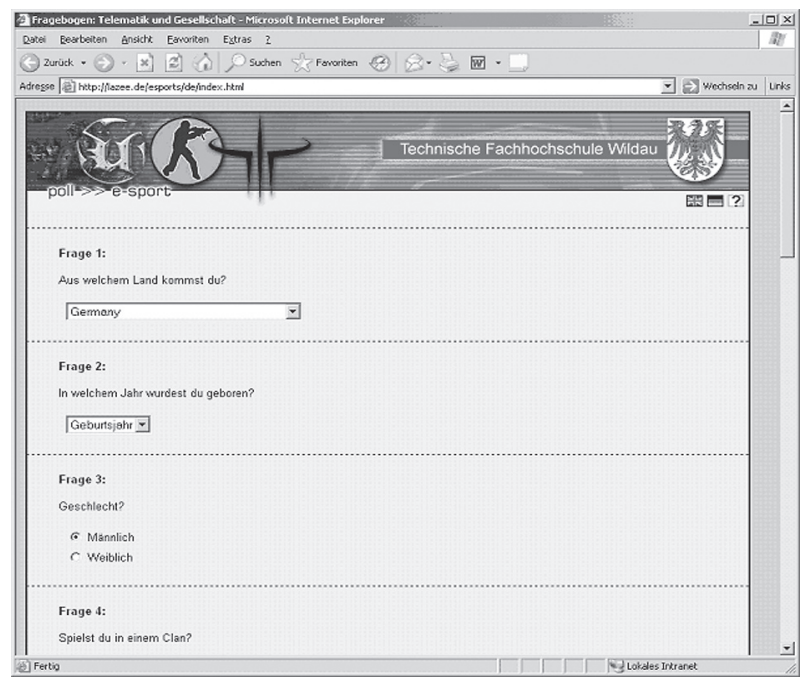

Bild 7: Screenshot der Umfrage

Es wurden 13 Fragen gestellt, die einem kurzen Interview entsprechen, um einen groben Überblick der wichtigsten Informationen zu erhalten. Dabei wurden die Fragen so gestaltet, dass sie später statistisch möglichst gut auswertbar sind. Da es im Internet eine Flut von Umfragen, Marktforschungen und auszufüllenden Formularen gibt, haben wir versucht den Bogen schnell ausfüllbar zu gestalten, da nach unseren Einschätzungen unsere Zielgruppe wenig Motivation hat sich lange mit einem Fragebogen aufzuhalten.

Um die Umfrage publik zu machen, bedienten sich die Autoren dieser Arbeit verschiedener Internetforen die hauptsächlich von Spielern besucht werden (http://forum.counterstrike.de/bb/, http://www.esl-europe.net/de/forum/, http: //www.piranho.com/home/boards), einem Hinweis auf einer privaten Webseite sowie verstärkt dem Medium IRC (Internet Relay Chat), welches als ein Hauptkommunikationskanal für Spieler verschiedener Richtungen und Herkunft benutzt wird. Auf diese Weise wurden 319 Umfragen gesammelt. Die effektiv verwertbare Anzahl von Umfragen (nach Entfernung von versehentlich doppelt abgegebenen Ergebnissen und mutwilligen Falschangaben) belief sich auf 301. Durch Unterscheidung der bei der Speicherung der Umfrage mitprotokollierten IP Adressen wird davon ausgegangen, dass 301 Personen an der Umfrage beteiligt waren.

Die ersten drei Fragen bezogen sich auf Herkunftsland, Geburtsjahr und Geschlecht.

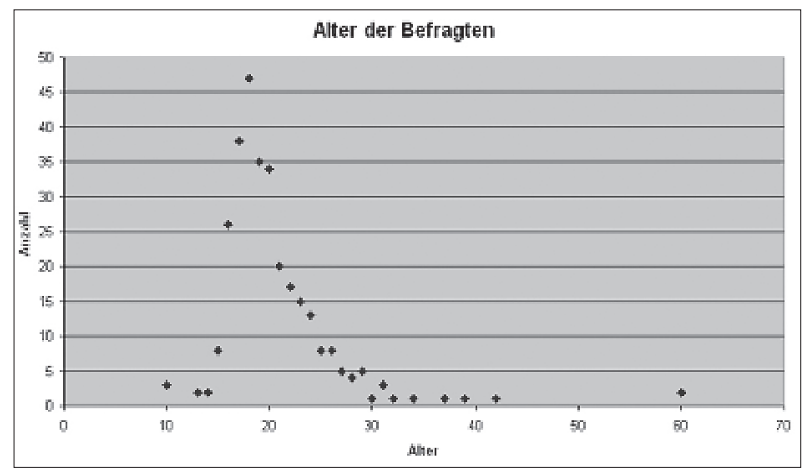

Bild 8: Altersverteilung 
Der Großteil der Umfrageteilnehmer war zwischen 16 und 21 Jahre alt, $92 \%$ waren männlichund $8 \%$ weiblich. Unsere Umfrage erreichte hauptsächlich deutsche Personen.

Die darauf folgenden Fragen beschäftigten sich tiefer mit dem Thema E-Sport. Es wurde dabei davon ausgegangen, dass die Befragten bereits mit den Begriffen vertraut sind und das Feedback, welches gegeben wurde hat auch gezeigt, das es keine Schwierigkeiten beim Verstehen der Fragen gab. Der folgende Abschnitt wird die Fragen auflisten und erklären sowie die gesammelten Ergebnisse darstellen.

Frage 4: Spielst du in einem Clan? (ja/nein)

Hier wurde versucht herauszufinden, wie groß der Anteil der organisierten Spieler ist, also Jenen, die in Clans oder Gilden sich mit anderen Spielern zusammengeschlossen haben. $47 \%$ der Befragten sind Mitglied in einem Clan oder einer clanähnlichen Gemeinschaft, $53 \%$ sind clanlos.

Frage 5: Wie viele Stunden bist du ungefähr täglich mit dem Spielen oder/und Organisieren von OnlineSpielen beschäftigt? (bis zu 1 Stunde/2-4 Stunden/4-6 Stunden/mehr als 6 Stunden) Das Ergebnis dieser Fragen war eine durchschnittliche Spieldauer von ca. 2,5 Stunden pro Tag unter allen 301 Befragten. Die genaue Verteilung war wie folgt:

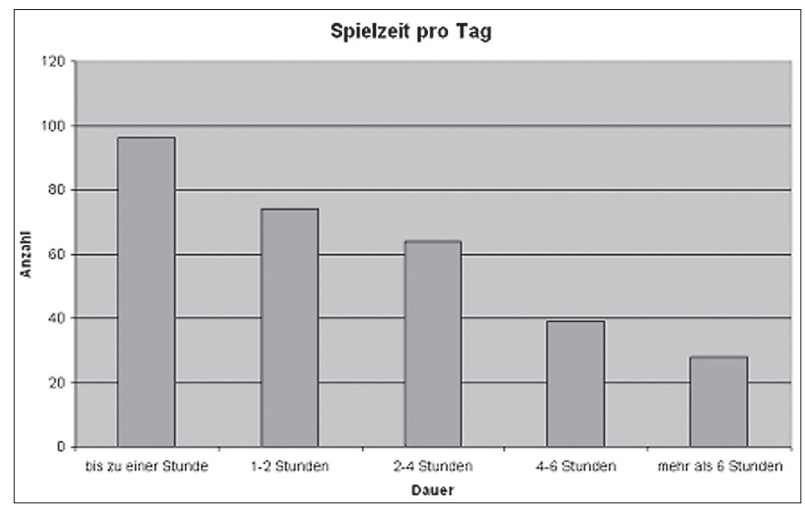

Bild 9: Tägliche Spielzeit

Frage 6: Welches Spiel spielst du am häufigsten online? (Auswahl einer Liste derzeit populärer Online Spiele) Es wurde versucht, eine Liste der zurzeit bekanntesten Online Spiele zu erstellen und die Teilnehmer der Umfrage daraus wählen zu lassen. Falls der Befragte sein favorisiertes Spiel nicht in der Liste auffinden konnte, konnte er ein Feld mit einer freien Texteingabe ausfüllen. Die Tatsache, dass 50 Nennungen für „anderes Spiel“ vorliegen und dass eine Vielzahl von Spielen im Feld mit der freien Texteingabe angegeben wurde, spiegelt die große Diversität auf dem Online Spielemarkt wider. Viele der alternativen Spielenennungen waren so genannte „Browser Games", welche man direkt über den Webbrowser nach einer meist kostenlosen Anmeldung spielen kann. Als das beliebteste Spiel zeichnete sich eindeutig „Counter-Strike 1.6" ab, welches nun mehr seit bereits 5 Jahren auf dem Markt ist. Es gehört auch zur Gruppe der Egoshooter, welche 61 \% aller Nennungen ausmachten. Nahezu alle genannten Egoshooter haben taktische Elemente, was den besonderen Reiz auszumachen scheint. $15 \%$ der genannten Spiele kann man im Bereich „Rollenspiele“ einordnen, Echtzeitstrategiespiele sind mit $5 \%$ vertreten. Der Bereich der „anderen Spiele“ teilt sich in Sportspiele, Strategiespiele, Rollenspiele und weitere Egoshooter auf.

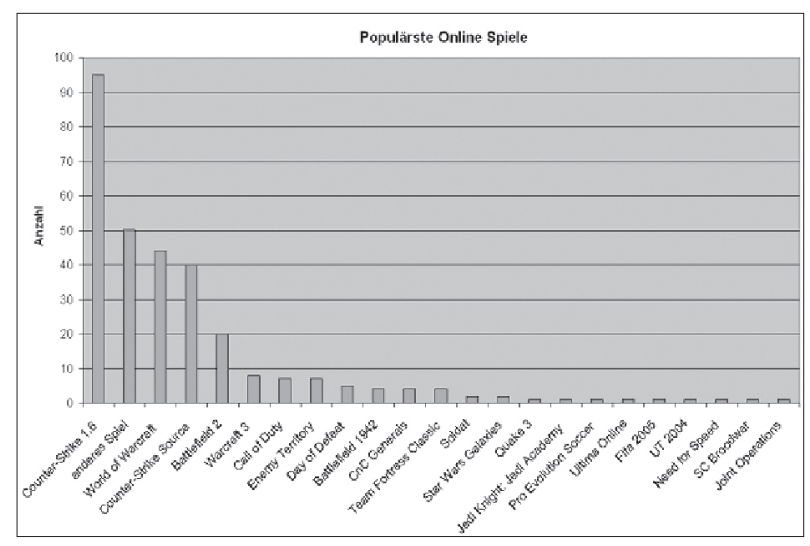

Bild 10: Populärste Online-Spiele

Frage 7: Spielst du in einer Liga? (ja/nein)

Die Frage zielte darauf ab, diejenigen Clanspieler oder Einzelspieler abzugrenzen, die in einer Liga gegen andere Spieler antreten und um Platzierungen oder Preisgelder spielen. Man könnte diese Gruppe auch als den Teil der Befragten bezeichnen, die am aktivsten am E-Sport teilnehmen. $35 \%$ der Befragten gaben an, in einer Liga mitzuspielen, $65 \%$ spielen in keiner Liga. Dabei ist anzumerken das bestimmte Online Spiele keine Eigenschaften mit sich bringen die ein Ligaspiel möglich machen sondern teilweise auch ein spielinternes Ranking der Spieler oder Clans/Gilden bieten (z. B. viele Rollenspiele).

Frage 8: Wird dein Clan gesponsort? (ja/nein) $22 \%$ der Befragten gaben an, einen Sponsor für ihren Clan zu haben, was $46 \%$ aller Clanspieler dieser Umfrage ausmacht. Wenn die Angaben wahrheitsgemäß waren, werden also knapp die Hälfte aller Clanspieler in Form von Geld, Hardware oder Dienstleistungen von Firmen oder Privatpersonen unterstützt.

Frage 9: Hast du selbst schon Geld verdient mit dem Organisieren bzw. Spielen von Online-Spielen? (ja/nein) Die Frage richtete sich an alle Teilnehmer, die schon einmal Geld verdient haben durch Gewinnen von Turnieren, regelmäßige Gelder von Sponsoren oder Bezahlung für Teilnahme oder Organisation von Spielen oder E-Sport Veranstaltungen. $16 \%$ der Befragten haben bereits Geld mit diesen Tätigkeiten verdient, $84 \%$ bisher nicht.

Frage 10: Könntest du dir vorstellen, deinen Lebensunterhalt mit eSports zu verdienen? (ja/nein)

Hier sollte sich zeigen, ob die Spieler ihr Hobby auch zum Beruf machen oder lieber das Spielen als Nebentätigkeit weiterführen würden. $32 \%$ haben Interesse am hauptberuflichen Spielen, $68 \%$ waren dagegen.

Frage 11: Verfolgst du regelmäßig die Spiele bekannter Clans oder Spieler? (ja/nein)

Ähnlich wie bei bereits etablierten Sportarten, wie Fußball oder Basketball, gibt es in der E-Sport Szene bekannte Spieler oder Stars, Fans und auch Spielübertragungen. Bisher werden die Spiele über Livestreams im Internet oder durch Zuschauermöglichkeiten die das Spiel selbst bietet übertragen, der TV Sender GIGA zeigt in seiner täglichen E-Sports Sendung Spitzenspiele auch im Fernsehen. Des Weiteren gibt es unzählige Webseiten die über die Spiele der Spitzenclans berichten. 31 \% der Umfrageteilnehmer verfolgen laut ihren Angaben regelmäßig Spiele bekannter Clans, $69 \%$ antworteten mit nein. 
Frage 12: Ist dein Freundeskreis im Onlinebereich oder im „wahren Leben“ größer?

Frage 13: Welche Freunde davon sind dir wichtiger? („wahres Leben“ / online / beide gleich)

Die beiden Fragen sollten die soziale Stellung von Freunden im „wahren Leben“ und im „virtuellen Leben“ online beleuchten. $81 \%$ der Teilnehmer gaben an, einen größeren Freundeskreis im „wahren Leben“ zu besitzen, $19 \%$ haben mehr Freunde im Online Bereich. Allerdings gibt es hier auch viele Überschneidungen, einige Teilnehmer der Umfrage wiesen uns darauf hin, dass es durchaus gemischte Verhältnisse von Online- und „wahrem“ Leben gibt:

- „Meine Freunde im wahren Leben sind zu 90\% die gleichen wie im Onlinebreich."

- „Onlinefreunde können auch zu Freunden im ,wahren Leben' werden."

- „Viele von meinen ,Onlinefreunden' sind meine ,Reallifefreunde' oder eher andersrum. Wir kennen uns alle privat, und zocken zusammen."

Die Frage nach der Wichtigkeit der Freunde aus dem realen Leben und dem Online Leben sollte eine Gewichtung dieser sozialen Beziehungen erkennbar machen. $81 \%$ der Befragten sind die Freunde aus dem realen Leben wichtiger, $5 \%$ bevorzugen ihre Online Freunde und $14 \%$ machen keinen Unterschied zwischen Freunden aus der Onlinewelt und dem wahren Leben. Auch zur Frage der Wichtigkeit folgten Kommentare der Befragten:

- „Da ich den Großteil meiner ,Onlinefreunde' schon seit Ewigkeiten kenne, sind die mir auch ans Herz gewachsen."

- „[...] sind mir alle gleich wichtig, ich mach da keine Unterschiede... ich kenn die ausm Clan beispielsweise schon seit 2 Jahren... ich treff mich mit denen 2 mal im Jahr mindestens... also es gibt da kaum Unterschiede... zwar sieht man sie weniger aber das Verhältnis ist gleich [...]"

Die 14. Frage war keine Frage an sich sondern bot Platz für die oben bereits angeführten Kommentare und Meinungen. Hier haben einige Teilnehmer auch die Gelegenheit genutzt um ihre persönliche Meinung zum Thema E-Sport zu äußern:

- „Persönlich finde ich, dass E-Sport nicht wirklich eine Disziplin ist, die etwas mit Sport zu tun hat. Schön, wenn es Leute gibt die damit Geld machen können. Ich würde es nicht wollen."

- "E-Sport ist ein Hype"

- „E-Sport ist meiner Meinung nach kein Sport und sollte auch nicht in diesen Status erhoben werden."

- „E-Sport ist meiner Meinung nach eine der dämlichsten Erfindungen, die es gibt. Spiele sind kein ,Sport'."

- „E-Sport ist ein Wunschtraum irgendwelcher Leute die besser mal vor die Tür gehen sollten."

- „Fordert mehr E-Sport in der Gesellschaft!“

- „E-Sport ist ne Leidenschaft"

- „der E-Sport wird eines Tages sicherlich in .de so groß sein wie in Japan und den andern asiatischen Ländern, irgendwann wird dieses auch als Beruf angesehen"

- „pro-Gaming macht die Spiele kaputt, sobald das Gewinnen im Vordergrund steht, bleibt der Spass mehr und mehr auf der Strecke..."

\section{Schlussfolgerungen aus den Ergebnissen der Befragung}

Um repräsentative Ergebnisse $z u$ erhalten, welche die Gesamtheit aller Spieler berücksichtigt, wäre sicher eine komplexer angelegte Umfrage und ein weitaus längerer Zeitraum nötig (Umfrage lief zwei Tage) gewesen. Jedoch ist es auch möglich aus diesem Ausschnitt von Spielern einige Erkenntnisse und Tendenzen abzulesen.

Die Altersübersicht zeigt, dass der Großteil der Teilnehmer im Bereich von 16 bis 21 Jahren liegt, jedoch das Feld der Spieler sich auch breiter in den jüngeren und älteren Bereich fächert. Mit 2,5 Stunden durchschnittlicher Spielzeit pro Tag sind sie bereit, einen relativ großen Teil ihrer Freizeit für ihr Hobby zu opfern. Das die teamspielfähigen Onlinespiele in der Häufigkeitsverteilung der gespielten Titel an erster Stelle stehen scheint einen besonderen Reiz beim Zusammenspiel mit anderen Menschen aufzuzeigen, das Egoshooter an erster Stelle stehen ist eher darauf zurückzuführen das es in diesem Bereich die meisten und auch ersten onlinefähigen Titel auf dem Markt gibt. Rollenspiele, die einen erhöhten Zeitaufwand benötigen, sind schwächer vertreten.

Die Tatsache, dass knapp die Hälfte aller befragten Spieler sich in Clans oder clanähnlichen Gemeinschaften zusammenfinden, lässt auf einen erhöhten Spielspaß bei Verknüpfung des Spiels mit einer sozialen Komponente schließen.

Der Anteil der Ligaspieler mit 35 \% deutet darauf hin, dass neben den Gelegenheitsspielern ein gutes Drittel der Befragten sich in einem organisierten Wettkampf mit anderen Spielern messen wollen und demnach zu aktiven E-Sportlern gezählt werden können.

Relativ überraschend war die Tatsache, dass $46 \%$ aller Clanspieler angaben, eine Form von Sponsoring in ihrem Clan zu erfahren. Es wird angenommen, dass dieses Sponsoring hauptsächlich in Form von Dienstleistungen und Bereitstellung von Spieleservern umgesetzt wird und eher in seltenen Fällen direkt Geld an die Spieler bezahlt wird.

Das wird auch deutlich bei der Frage nach bisherigen Geldeinnahmen durch Onlinespiele, bei der $16 \%$ angaben, schon einmal Geld mit ihrem Hobby verdient zu haben. Ein größerer Teil der Befragten, mit 32 \%, könnte sich jedoch vorstellen, sogar den Lebensunterhalt mit E-Sport zu verdienen, was sich fast mit der Anzahl der Ligaspieler deckt. Fast dieselbe Größe haben mit 31 \% die Anzahl der Spieler, die regelmäßig Spiele bekannter Clans oder Spieler verfolgen und ein Vergleich mit den Daten der Ligaspieler hat ergeben, dass Teilnehmer die in einer Liga spielen auch größtenteils jene sind, die aktiv das Spielgeschehen in den bekannteren Bereichen der E-Sportszene verfolgen.

Die eher sozial bezogenen Fragen zum Thema Freundeskreis online und offline ergaben, dass ein Großteil der Umfrageteilnehmer den Menschen im wahren Leben mehr Bedeutung zurechnet und dort auch den größten Freundeskreis hat. Jedoch gibt es hier auch Überschneidungen, da mit Menschen aus dem Alltag auch Online kommuniziert wird und weil aus Bekanntschaften die im Onlinebereich entstehen auch in der realen Welt soziale Beziehungen entstehen. Einige der Teilnehmer wiesen uns darauf hin, dass der Unterschied zwischen den Gruppen Online- und Offlinefreunde als nicht sehr signifikant darzustellen sei. 


\section{Schlusswort}

Es wurde gezeigt, dass der E-Sport ein großes Potential besitzt und für das Marketing von Unternehmen unterschiedlicher Branchen ein gutes Mittel ist, die Zielgruppe der „Heranwachsenden“ zu erreichen. Der E-Sport hat in weniger als zehn Jahren eine beachtliche Entwicklung durchgemacht. An den „World Cyber Games“ nehmen schon fast so viele Länder teil, wie an den Olympischen Spielen. Deutschland steht zwar momentan noch in den internationalen Wettkämpfen relativ gut da, doch darf hier auch von staatlicher Seite der Trend nicht verschlafen werden. Urteile wie in dem Abschnitt „Vergleich zwischen E-Sport und reellem Sport" sind nicht nur unnötig, sondern auch schädlich, da sie die öffentliche Wahrnehmung beeinflussen und so die Entwicklung in Deutschland unter Umständen stagnieren lassen.

Langfristig ist ein Erfolg, ähnlich wie in Südkorea, auch hier in Deutschland durchaus denkbar. Aber es ist nicht anzunehmen, dass dies so extrem geschehen wird, wie dort. Europa wandelt sich nicht so schnell. Dinge brauchen hier Zeit sich zu entwickeln.

Mit der Gründung des „Deutschen eSport Bundes“ wurde ein Schritt in die richtige Richtung getan, E-Sport in der öffentlichen Gesellschaft besser zu repräsentieren.

Wir möchten uns abschließend bei all jenen bedanken, die an unserer Umfrage teilgenommen haben und unsere Fragen teilweise so ausführlich beantworten haben. Außerdem gilt unser besonderer Dank unserem Dozenten Prof. Dr. Bernd Eylert, welcher uns in seinem Seminar „Telematik und Gesellschaft" ermöglicht hat, ein Thema zu behandeln, welches uns beide auch privat seit einiger Zeit beschäftigt und deshalb auch besonderen Spaß macht.

\section{Fußnoten}

(1) Interleaving ist ein Verfahren, welches durch Umsortierung der Übertragungsbits Burstfehler verteilt

\section{Glossar}

Aimbot : Ein Schummelprogramm, welches Gegner automatisch anvisiert.

BPjM: Bundesprüfstelle für jugendgefährdende Medien

Cheatprogramme: Programme, welche es erlauben in Spielen zu schummeln

Counter-Strike: derzeit bekanntester Taktik - Egoshooter

Echtzeitstrategiespiel: Spiel, in welchem man, meist in isometrischer Ansicht, verschiedene Einheiten in Echtzeit befehligt

Egoshooter: ein Spiel, welches eine virtuelle Welt in einer 3DEgoansicht darstellt und meistens ein Teamspiel unterstützt

fatal1ty: Jonathan Wendel, einer der ersten amerikanischen Progamerstars

Gameserver: Ein Rechner im Internet, auf welchem dedizierte Serverprogramme (meist) unterschiedlicher Spiele laufen

IRC: Internet Relay Chat, eines der ältesten und größten Chatsysteme

Lags: Sekundenaussetzer bei einem Onlinespiel aufgrund mangelhafter Datenkommunikation
LAN-Party: Party, auf welcher bis zu mehrere Hundert PCs lokal vernetzt werden

MMORPGs: Massive Multiplayer Online Rollplay Games, Spiele bei welchen oft Hunderte Spieler auf einem Server an einem virtuellen Rollenspiel teilnehmen

Progamer: professioneller Spieler

Quake III: sehr populärer Egoshooter, welcher meist „Mann gegen Mann" gespielt wird

Starcraft: das derzeit noch populärste Echtzeitstrategiespiel in Südkorea

USK: Unterhaltungssoftware Selbstkontrolle, freiwillige Selbstkontrolle der Spieleindustrie zur Einstufung der Software in bestimmte Altersgruppen

Wallhack: Ein Schummelprogramm, welches es erlaubt in einem Egoshooter durch Wände zu schauen

WCG: World Cyber Games, jährlich stattfindender, größter, ESport Event

\section{Literatur/Quellen}

[WI05] 29.Juni 2005, de.wikipedia.org/wiki/Esports

[LI05] 29.Juni.2005, Lietzkow, Seite 1, www.esportforum.com/ vortraege/Vortrag_Lietzkow.pdf)

[WI205] 30.Juni 2005, de.wikipedia.org/wiki/E-sport

[KR05] 30.Juni 2005, www.korea.net/news/news/ newsView.asp?serial_no=20041217018\&part=108 $\S$ SearchDay $=$

[FAT05] 1. Juli.2005, www.fatal1ty.com/news/ ?type=PR\&ID $=15$

[KI05] 1. Juli 2005, Kim, http://www.esportforum.com/ vortraege/Vortrag_Kim.pdf

[MI05] 1. Juli 2005, Militz, http://www.esportforum.com/ vortraege/Vortrag_Militz.pdf

[KO05] 1.Juli 2005, Korte, http://www.esportforum.com/ vortraege/Vortrag_Korte.pdf, Seite 10

[KO205] 1. Juli 2005, Korte, http://www.esportforum.com/ vortraege/Vortrag_Korte.pdf, Seite 11

\section{Autoren}

B. IC. Sc. Henri Schmidt

Technische Fachhochschule Wildau lazeee@gmail.com

B. IC. Sc. Stefan Lehmann

Technische Fachhochschule Wildau stefan@4pmail.de 